\title{
The Milli-Arc-Second Structure Imager, MASSIM: A new concept for a high angular resolution X-ray telescope
}

\author{
G. K. Skinner ${ }^{a, b, c}$, Z. Arzoumanian ${ }^{a, c, d}$, W. C. Cash ${ }^{e}$, N. Gehrels ${ }^{c}$, K. C. Gendreau ${ }^{c}$, P. \\ Gorenstein $^{f}$, J. F. Krizmanic ${ }^{a, c, d}$, M. C. Miller ${ }^{b}$, J. D. Phillips ${ }^{f}$, R. D. Reasenberg $f$, C. S. \\ Reynolds $^{b}$, R. M. Sambruna ${ }^{c}$, R. E. Streitmatter ${ }^{c}$, D. L. Windt ${ }^{g}$ \\ ${ }^{a}$ CRESST, Greenbelt Road, Greenbelt, MD 20771 \\ ${ }^{b}$ University of Maryland, College Park, MD 20742 \\ ${ }^{c}$ NASA-GSFC, Greenbelt Road, Greenbelt, MD 20771 \\ ${ }^{d}$ USRA, 10211, Wincopin Circle Suite 500, Columbia Md 21044 \\ ${ }^{e}$ University of Colorado, Boulder, CO 80309 \\ ${ }^{f}$ Smithsonian Astrophysical Observatory, 60, Garden Street, Cambridge, MA 02138 \\ ${ }^{g}$ Reflective X-ray Optics, LLC, 1361 Amsterdam Ave, Suite 3B, New York, NY 10027
}

\begin{abstract}
MASSIM, the Milli-Arc-Second Structure Imager, is a mission that has been proposed for study within the context of NASA's Astrophysics Strategic Mission Concept Studies program. It uses a set of achromatic diffractiverefractive Fresnel lenses on an optics spacecraft to focus 5-11 keV X-rays onto detectors on a second spacecraft flying in formation $1000 \mathrm{~km}$ away. It will have a point-source sensitivity comparable with that of the current generation of major X-ray observatories (Chandra, XMM-Newton) but an angular resolution some three orders of magnitude better. MASSIM is optimized for the study of jets and other phenomena that occur in the immediate vicinity of black holes and neutron stars. It can also be used for studying other astrophysical phenomena on the milli-arc-second scale, such as those involving proto-stars, the surfaces and surroundings of nearby active stars and interacting winds.

We describe the MASSIM mission concept, scientific objectives and the trade-offs within the X-ray optics design. The anticipated performance of the mission and possible future developments using the diffractiverefractive optics approach to imaging at X-ray and gamma-ray energies are discussed.
\end{abstract}

Keywords: X-ray. Gamma-ray, Imaging, Fresnel, Formation Flying

\section{INTRODUCTION}

MASSIM (Milli-Arc-Second Structure Imager, Fig. 1) is a proposed mission in the domain of ultra-high angular resolution X-ray astronomy, which is a combination of terms that do not usually go together. It is ironic that the angular resolution of current X-ray instrumentation is no better than that routinely attained at optical wavelengths, even though the diffraction limit is a thousand or more times lower. In the gamma-ray band, where the diffraction limit is still lower, the situation is even more extreme and the present generation of instruments have resolution $\sim 10$ arc minutes or poorer - not very much better than the angular size of the full moon!

The technology proposed for MASSIM allows 2-3 orders of magnitude improvement in angular resolution over that of the Chandra mission which represents the present state of the art. For comparison, Galileo revolutionized astronomy with telescopes that improved angular resolution by about a factor of six over that of the naked eye. ${ }^{1}$ The diffractive/refractive lens technology used for MASSIM will allow the X-ray band to take its logical place at the forefront of ultra-high angular resolution astronomy. The technology is capable of extension both to larger systems and to higher (hard X-ray and gamma-ray) energies, allowing micro arc second resolution.

Further author information: (Send correspondence to G.K.S.)

G.K.S..: E-mail: gkskinner@nasa.gov, Telephone: 13012861350

Space Telescopes and Instrumentation 2008: Ultraviolet to Gamma Ray, edited by Martin J. L. Turner, Kathryn A. Flanagan, Proc. of SPIE Vol. 7011, 70110T, (2008) · 0277-786X/08/\$18 · doi: 10.1117/12.789568 


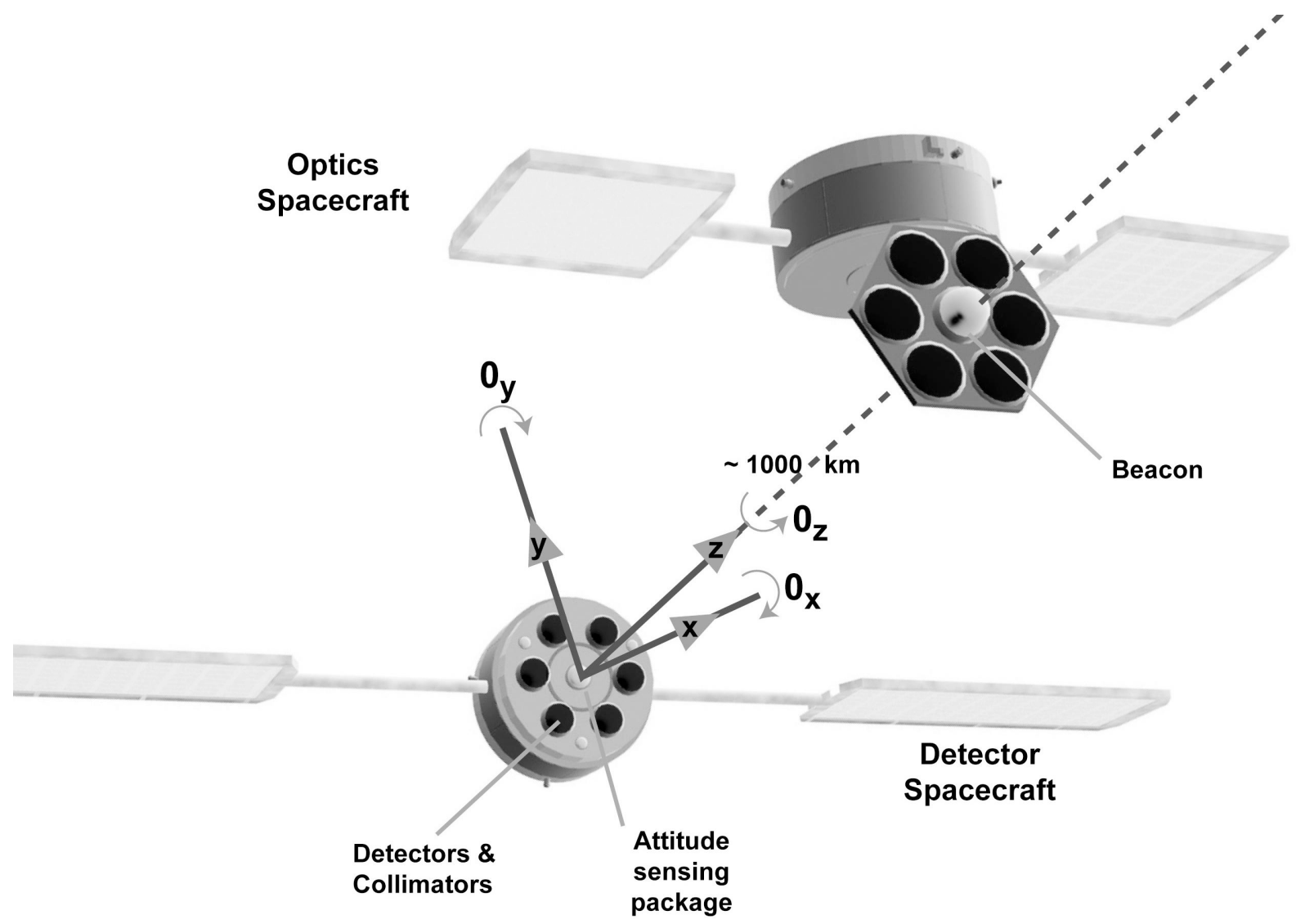

Figure 1. The MASSIM mission concept will use a technology new to X-ray astronomy to improve angular resolution by up to 3 orders of magnitude. An optics spacecraft carrying an array of diffractive-refractive lenses focuses X-rays onto detectors on a spacecraft $1000 \mathrm{~km}$ behind.

The MASSIM mission was proposed in response to NASA's "Advanced Mission Concept Studies" opportunity in November 2007. Although it was not selected for study, the concept and related ideas are continuing to be developed in anticipation of other opportunities.

\section{SCIENTIFIC OBJECTIVES}

The capabilities of MASSIM are such that it can impact a wide range of important scientific topics from stars and planets to more exotic objects such as supermassive black holes (SMBHs) and quasars. Here, we briefly survey the science goals of MASSIM with an emphasis on contributions to our understanding of phenomena in the vicinity of black holes and in particular of jets.

\subsection{Jets from black holes - stellar to supermassive}

Jets are ubiquitous in astronomy. With improving instrumentation, jets are being discovered in more and more circumstances, from recently formed pulsars and young proto-stellar systems to old X-ray binaries and the dying stars that lead to $\gamma$-ray bursts, and from stellar mass black holes of a few solar masses $\left(\sim \mathrm{M}_{\odot}\right)$ to active galactic nuclei (AGN) containing SMBHs of more than $10^{9} \mathrm{M}_{\odot}$. Jets are intimately linked to numerous aspects of the origin, structure, evolution and destiny of the Universe - the heating of the interstellar and intergalactic medium by AGN jets is widely believed to be an important process in regulating galaxy growth ${ }^{2}$. 
Jets include the largest single objects in the Universe (several $\mathrm{Mpc}$ ) but are produced in regions so compact that they are difficult to study. It is still uncertain where and how the matter in jets is accelerated - most successful models suggest gentle hydromagnetic acceleration, but there is little direct observational evidence for or against these ideas. Even the basic nature of the accelerated matter is unclear - are black hole jets electronpositron or normal electron-proton plasmas? It seems likely that jets are Poynting flux dominated within the acceleration region but it remains an open question where a jet becomes matter dominated ${ }^{3}$. A question of fundamental importance concerns the primary energy source for these jets -are they energized by the inner accretion flow $^{4-6}$ or the magnetic extraction of spin energy from the black hole ${ }^{7,8}$ ?

Radio VLBI (very long baseline interferometry) images with milli-arcsecond resolution have been instrumental in our attempts to understand black hole jets. VLBI has revealed blobs of radio-emitting plasma ejected at high speed both in the jets of AGN involving SMBHs and in those in microquasars in our own galaxy, which seem to be their stellar mass black hole analogs. ${ }^{9}$ Often the apparent ejection speeds are superluminal, proving that these flows are highly relativistic. In nearby AGN (e.g. M87), VLBI observations track the jet down to scales of less than $10^{2}$ Schwarzschild Radii, ${ }^{10}$ demonstrating that collimation and (probably) acceleration occurs on these scales.

MASSIM's milli-arc-second angular resolution will enable us to directly image the immediate BH environment on VLBI scales, where jets are accelerated and collimated. Its sensitivity in an energy range (4.5-11 keV), where absorption by dust and gas is negligible, will allow a direct peek into regions virtually inaccessible at other wavelengths and contains the all-important Fe K lines.

Combining X-ray images from MASSIM with VLBI maps will allow us, for the first time, to separately determine the magnetic field strengths and particle densities/energies of milli-arcsecond scale jets (via standard synchrotron theory). Measurements of these basic physical quantities are crucial if we are to test jet acceleration/collimation models at a level of detail beyond the crude consistency tests possible today. Combined with secondary indicators of jet power (e.g., the power needed to inflate large scale cavities in the surrounding medium) these measurements will allow powerful tests of the particle content (pair plasma vs normal plasma) and Poynting flux content of the jets. ${ }^{11,12}$

The majority of AGN are radio quiet, ${ }^{13}$ failing to display powerful radio jets. Milli-arcsecond jets are, however, commonly found in radio-quiet Seyfert nuclei using VLBI. The reason why jets cannot be fully fledged in Seyferts, despite the presence of accretion onto a $\mathrm{SMBH}$, is still unknown. It may involve aspects of the black hole itself (e.g., its spin rate and/or the magnetic flux threading the black hole) or a foetal jet may be quenched by its environment. MASSIM will image the closest and best resolved radio jets in Seyferts (e.g. III Zw 2, Ref 14) and allow us to derive quantitative information on the jet properties and environment, thus testing these scenarios.

The closest SMBH to us is at our Galactic Center and continues to be a crucial test-bed for models of black hole accretion. The low-luminosity of this black hole despite the presence of surrounding gas (originating from stellar winds of the central star cluster) is interpreted in terms of a Radiatively-Inefficient Accretion Flow (RIAF). ${ }^{15}$ MASSIM will enable us to resolve X-ray emission from the accretion flow allowing unprecedented tests of the RIAF models. The importance of this is highlighted by the realization that most SMBHs in the Universe accrete in this mode.

\subsection{MASSIM as a multipurpose X-ray observatory}

MASSIM will be a highly capable, multi-purpose high angular resolution X-ray observatory and will have impact across astrophysics (see Figs. 4,5). In addition to its study of jets, the angular resolution and the energy band of MASSIM are ideal for using the fluorescent Fe lines to explore the parsec-scale structure of AGN, including the molecular torus often discussed in the context of AGN unification. Within our own galaxy, MASSIM will study pulsar wind nebulae. MASSIM observations of the Crab Pulsar will allow highly resolved images of the ultra-relativistic (Lorentz factor $\sim 10^{6}$ ) MHD wind that is believed to be accelerated by this rotating magnetized neutron star. ${ }^{16}$ Chandra has observed the (arc-second scale) shock where this wind collides with the surrounding matter, ${ }^{17}$ but MASSIM resolution is required to study the evolution of this wind closer to the pulsar's light cylinder. 
Another example is MASSIM's ability to resolve coronal emission in nearby stars. This would give us our first resolved view of any stellar corona beyond the Sun, dramatically improving our understanding of magnetically active stars. It will image the emission from interacting winds in binary star systems, in which simulations suggest that instabilities lead to complex structures.

Finally, a prospect indicated by some recent unexpected discoveries is that of imaging protoplanetary-nebulae in X-rays. ${ }^{18-21}$

\section{MISSION CONCEPT}

The MASSIM mission concept involves two spacecraft, placed in the region of the L2 Langrangian point in a dual launch with a Delta $\mathrm{M}+(5,2)$ or Atlas 511 class launch vehicles. A drift away heliocentric orbit is a possible alternative. The two spacecraft will fly in formation with a separation of about $1000 \mathrm{~km}$ in such a way that radiation from the front, optics, spacecraft is focussed onto an array of detectors on the other spacecraft.

\subsection{The optics spacecraft}

The key that makes possible the great step forward offered by MASSIM is the use of transmissive lenses relying on a combination of diffraction and refraction. It is interesting that Fresnel zone-plates, closely related to the Phase Fresnel lenses now being considered, were noted as a possible alternative to Kirkpatrick-Baez reflective optics in the early days of X-ray imaging ${ }^{22}$ and that X-ray images of the sun obtained with a Fresnel zone-plate were reported in $1968 .{ }^{23}$ But it is reflective optics that has come to dominate high-energy astronomy, at least until now.

X-ray optics based upon grazing incidence reflection requires large areas of mirror surface to be very well polished, precisely figured and accurately aligned. Manufacturing constraints currently limit the angular resolution to little better than an arc-second. Refractive-diffractive optics operated in transmission circumvent these practical limitations - the tolerances necessary to achieve diffraction limited imaging are relatively easily achieved and as they are single 'thin' lenses, or contact-pairs of such elements, there are no issues of alignment of the optical components.

The refractive index of any material in the X-ray band differs slightly from unity, but the effect is small $(\delta=$ $\left.1-\mu \sim 10^{-4}-10^{-8}\right)$. As $\delta$ is normally positive, a purely refractive converging lens is concave. The edge thickness of a lens of diameter $d$ and focal length $f$ must be at least $t_{\min }=d^{2} /(8 f \delta)$. If a diameter of the order of $1 \mathrm{~m}$ is desired, either to have high collecting area or for good diffraction-limited angular resolution, then $t_{\min }$ is such that photoelectric absorption is too strong, even for large $f$. On the other hand Phase-Fresnel lenses are much thinner. They rely on the fact that focussing can be achieved if the phase of radiation that passes through a lens at a given radius is changed by an amount that need never be greater than $2 \pi$. The thickness of material needed to do this is $t_{2 \pi}=\lambda / \delta$. Because in the X-ray band $\lambda$ is so short, $t_{2 \pi}$ is small despite the minuteness of $\delta$ and for many combinations of material and $\lambda$ the absorption losses are low.

As a variant of the Fresnel zone-plate, Phase Fresnel lenses are intrinsically chromatic - their focal length is proportional to $\lambda^{-1}$. The lenses proposed for MASSIM are achromatic combinations of a Phase Fresnel lens and a refractive one (Fig. 2). Because of the absorption losses the refractive component is stepped like the diffractive one, though with very much coarser steps. In consequence the correction is approximate except at certain photon energies where the steps correspond to a phase difference that is a multiple of $2 \pi$.

Because $\delta$ is so small, constructional tolerances are enormously eased compared with those associated with reflective optics. Provided the focal length is sufficiently large, near diffraction-limited imaging can be achieved with low mass and at low cost. The use of Phase-Fresnel lenses and their related achromatic diffractive-refractive combinations for high energy astronomy has been proposed in the literature and many of their characteristics studied $^{24-39}$ but MASSIM is the first detailed mission concept that has resulted from this work.

Even an achromatic doublet has a limited bandwidth and MASSIM uses six lenses to cover the required 4.5-11 keV band (chosen to include the important 6-7 keV Fe lines). Each lens has a diameter of $1 \mathrm{~m}$ and has high efficiency, leading to an effective area of several thousand $\mathrm{cm}^{2}$. The way in which the bandpasses of the individual lenses combine to provide high effective area over the complete band is shown in Fig. 3. 


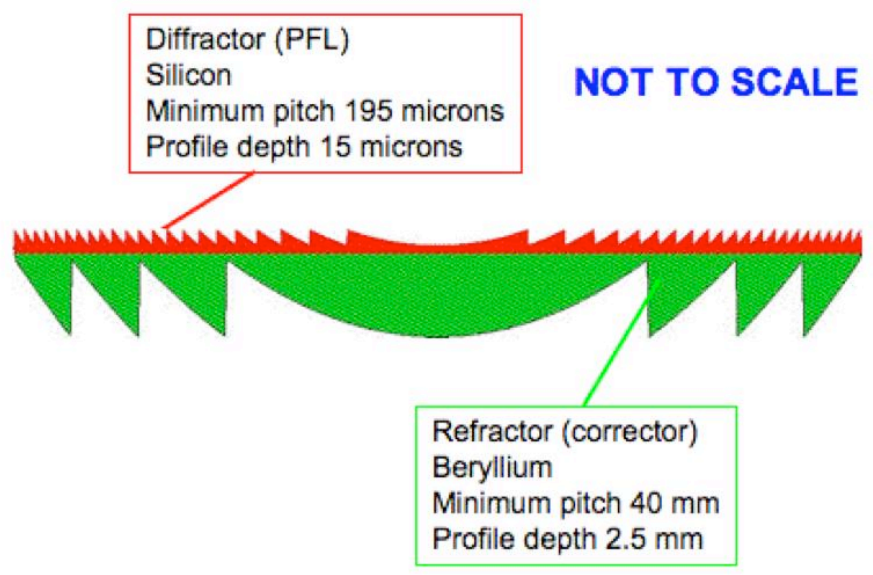

Figure 2. A diffractive-refractive achromat consists of a fine PFL (upper, brown) and a refractive lens (lower, green), which may itself be stepped on a much larger scale. Example materials and characteristic sizes are given for a $6 \mathrm{keV}$ lens with a focal length of $1000 \mathrm{~km}$. The diagram is indicative and not to scale - the $1 \mathrm{~m}$ diameter lens is only a few mm thick.

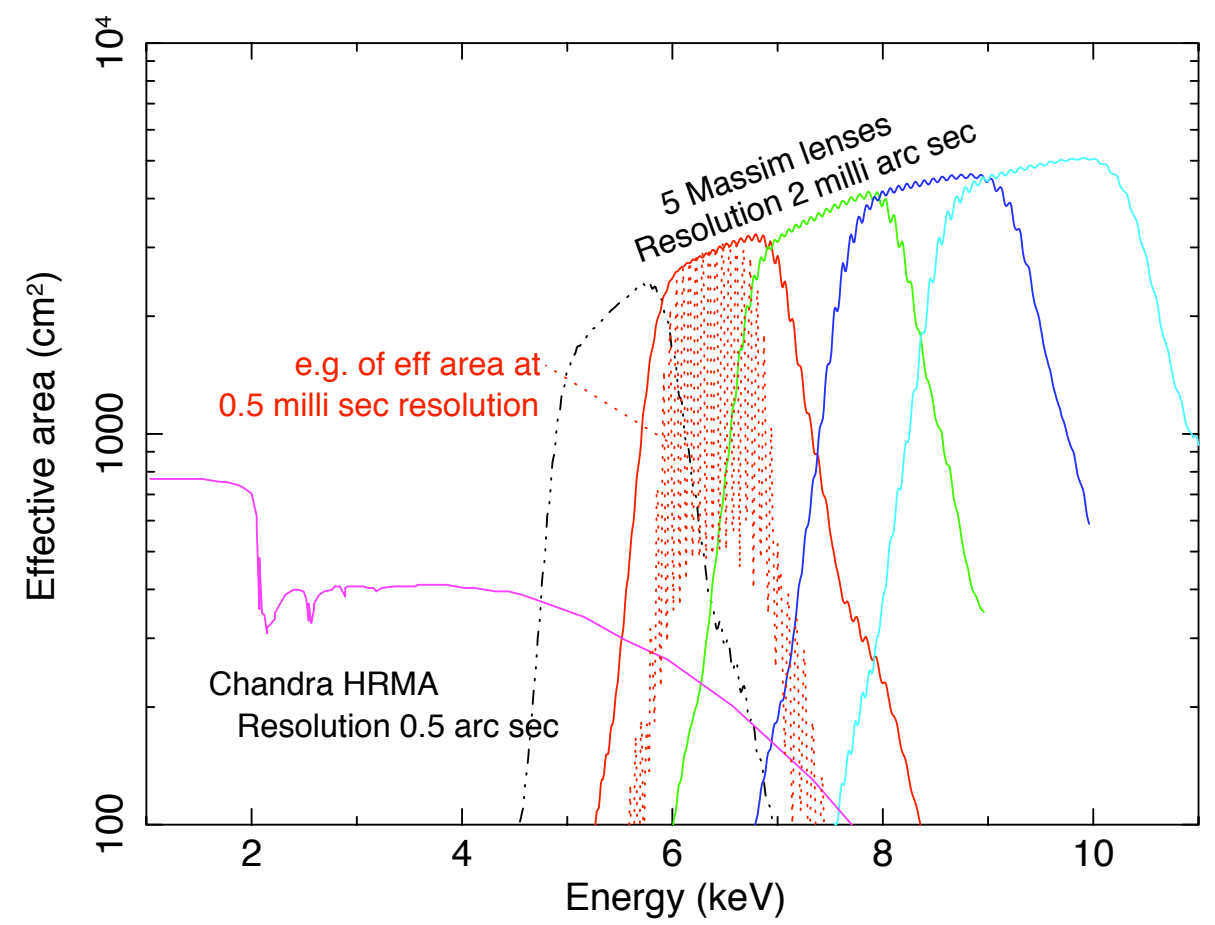

Figure 3. The effective area of an instrument with five 1-meter diameter diffractive-refractive lenses tuned to different energies. The effective area of the Chandra High Resolution Mirror Array is shown for comparison. The main curves correspond to the photons collected within a spot corresponding to 2 milli-arc-seconds but the angular resolution is considerably better (down to 0.5 milli-arc-seconds or better) at a comb of energies within the main pass band (e.g. dotted line). 
The refractive component of each lens is made of Beryllium since absorption is reduced for the needed thickness compared to other potential materials. For the much thinner diffractive component, the material can be chosen for ease of manufacture and candidate materials include silicon or plastic.

The effective area in Fig. 3 is based upon 5 lenses. A sixth station could be used to provide additional response around the Fe lines, to extend the energy range, or for some other options that are being studied, such as a constant pitch lens, analogous to a Talbot interferometer, that would provide lower effective area but over a wide bandpass. Another possible use of the sixth station would be for a lens of much shorter focal length that would allow a wide field of view to be obtained by moving the spacecraft closer together. This could be valuable for performance verification, for target finding in case of poorly known source location or for observation of lower surface brightness, larger scale, features.

An interesting possibility is the use of the opaque region around the lenses as an occulting disc. Sufficiently bright sources could be mapped on a larger scale and over a wide energy band by maneuvering the detector spacecraft across the shadow of the optics array. Again this mode would be useful for performance verification and target finding.

\subsection{The detector spacecraft}

The lenses form real images in a focal plane at a distance of $1000 \mathrm{~km}$. In this plane a second spacecraft will carry a series of large area detectors which can be based on existing technology. The performance of the CCDs used on Chandra, XMM, and Suzaku provide adequate energy resolution and a spatial resolution better than needed. A $500 \mathrm{~mm} \times 500 \mathrm{~mm}$ array of CCDs for each lens is baselined. With a focal length of $1000 \mathrm{~km}$ this corresponds to a field of view of about 100 milli-arc-seconds, so the instrument is optimized for the study of small compact regions of emission (more extended regions would anyway be of low surface brightness when examined at the MASSIM resolution). The aberration-free field of view of the lenses is very large, so larger detectors would increase the field of view accessible without scanning.

A collimator in front of each detector reduces the diffuse X-ray background. Its $\sim 1^{\circ}$ field of view will allow some radiation from a patch of sky around the optics spacecraft to reach the detector but background in the detectors due to diffuse background and sources in this region will be negligible compared with other background components, wheras the flux from the target source will be concentrated by a factor of $\sim 10^{5}$ by the lens. Radiation that has been focussed by a lens other than that directly in front of the detector will be recognizable by being of a different energy and will provide supplementary information outside the nominal field of view.

\subsection{Attitude Control and Formation flying}

The mission requires the formation flying of two spacecraft but in many respects the requirements are rather loose. For neither spacecraft is precision control of the attitude needed and the distance between the spacecraft is not critical. The crucial task for the control system is the determination and correction of the direction of the line of sight - the line joining a reference point at the center of the lens array to one at the center of the detector array. This 'pointing' direction must be controlled with enough precision to keep the target within the 100 milli-arc-second field of view and drift must be determined to better than a milli-arc-second in order to allow image reconstruction without degradation of the angular resolution.

A baseline concept for the pointing control system relies on very high precision star-trackers on the detector spacecraft viewing both a beacon at the center of the optics spacecraft and the background of stars behind it. In order to achieve the precision and bandwidth required, relatively bright reference stars must be used, leading to a need for a cluster of offset-able star trackers. The requirements on the sensors are well within the performance already achieved with the $14 \mathrm{~cm}$ star sensor on Gravity Probe B.

A continuous force is needed from thrusters on one of the spacecraft in order to maintain their relative positions in the presence of gravity-gradient forces and of differences in the accelerations due to radiation pressure, etc. Space-qualified Ion thrusters having the necessary performance have been identified for this and for repointing maneuvers. Based on a $1300 \mathrm{~kg}$ detector spacecraft and on 50 pointings per year over a 3 year mission, current thruster performance leads to a fuel requirement of approximately $100 \mathrm{~kg}$. 


\section{EXPECTED CAPABILITIES}

The expected capabilities of MASSIM are summarized in Table 1 and the effective area as a function of energy is shown in Fig. 3. The effective area is that for flux collected within a spot in the focal plane corresponding to an angular resolution of 2 milli-arc-seconds. At energies for which the steps in the refractive component of the lens correspond to a phase shift that is a multiple of $2 \pi$, the angular resolution will be much better. The dotted line indicates for one of the lens-detector systems how at certain energies within the design band a resolution of 0.5 milli-arc-seconds is achievable.

Fig. 3 also shows for comparison the effective area of the Chandra HRMA (High Resolution Mirror Array) that has an angular resolution of 0.5 arc-seconds. The effective area of MASSIM is so much greater than that of Chandra that the sensitivity is comparable despite the larger focal spot size.

Table 1. Baseline MASSIM characterics.

\begin{tabular}{|l|l|}
\hline Energy Range & $4.5-11 \mathrm{keV}$ \\
Focal length & $1000 \mathrm{~km}$ \\
Effective Area & $2000-4000 \mathrm{~cm}^{2}$ (inside 2 milli-arc-sec) \\
Angular Resolution & 2 milli-arc-seconds (Half Energy Width) \\
& $<0.5$ milli-arc-seconds (selected energies) \\
Field of view & 100 milli-arc-secs $(1)$ \\
Point source sensitivity & $8 \times 10^{-15} \mathrm{erg} \mathrm{cm}^{-2} \mathrm{~s}^{-1}(2)$ \\
\hline (1) Detector limited; $500 \times 500 \mathrm{~mm}$ assumed \\
+ possibility of wider field of view, lower resolution, option. \\
(2) $5 \sigma$ in $10^{5} \mathrm{~s}, 4.5-11 \mathrm{keV}$
\end{tabular}

\section{STATUS AND PROSPECTS}

Although MASSIM was not selected as one of the missions to be further studied in the context of the Advanced Mission Concept Studies, the proposing team is continuing work on this and related concepts.

A program of developing miniature lenses and testing them at the GSFC $600 \mathrm{~m}$ long beam test facility has been underway for several years and is continuing. The lenses are fabricated by colleagues at the University of Maryland using gray-scale technology with deep reactive ion etching. ${ }^{40,41}$ Although ground testing of a $1000 \mathrm{~km}$ focal length lens is impracticable, these miniature lenses are in many ways representative of larger ones and in many respects full size lenses would be easier to fabricate and less sensitive to manufacturing tolerances.

The number of grooves in the profile of a Phase Fresnel lens having radius $r$ and focal length $f$ at wavelength $\lambda$ (or photon energy $E$ ) is given by

$$
n=\frac{r^{2}}{2 f \lambda}=\frac{r^{2}}{2 f} \frac{h c}{E}
$$

The depth of the grooves of course depends only on the material and the wavelength and is independent of focal length. Consequently two lenses with focal lengths $1000 \mathrm{~km}$ and $100 \mathrm{~m}$ whose radii are in the ratio $\sqrt{10^{6} / 10^{2}}: 1$, or $1 \mathrm{~m}: 10 \mathrm{~mm}$, have the same number of grooves and can be identical except in radial scale. The miniature lenses that we are testing are based on this principle. Unfortunately, the scaling-down increases the aspect ratio of the groove structure to the point where manufacture becomes challenging. However the University of Maryland group have produced lenses up to $5 \mathrm{~mm}$ in diameter and with a focal length of $\sim 100 \mathrm{~m}$ at $8 \mathrm{keV}$. Testing of these lenses, both alone and in conjunction with refractive achromatic correctors, is on-going at GSFC.

One of the aspects of the MASSIM mission that would have been studied within the Advanced Mission Concept Studies context is that of formation flying and the associated navigation and pointing determination issues. Fortunately many of the MASSIM requirements are similar to those of the New Worlds mission, for which an AMCS study is now underway. Like MASSIM, New Worlds requires milli-arc-second level control of 
the direction in celestial coordinates of the line joining two spacecraft. ${ }^{42}$ For New Worlds, the planned separation between an 'occulter' spacecraft and one carrying a telescope is $70000 \mathrm{~km}$, compared with the $1000 \mathrm{~km}$ baselined for MASSIM. So in some respects the formation flying requirements for New Worlds are more challenging. The findings of this study will clearly be of direct relevance to MASSIM and similar concepts for Fresnel lens imaging at high energies.

The MASSIM design parameters were chosen based on particular scientific objectives and bearing in mind particular programmatic constraints. The same general concept is capable of being optimized in different ways. By going to longer focal lengths, or to smaller lenses, even better angular resolution would be possible, though at the expense of field of view in the one case and of sensitivity in the other. Although the energy band was chosen to be centered on the 6-7 keV Fe lines, the same technology can be used over a wide range of energies. At high energies longer focal lengths become preferable, but the diffraction limit allows better resolution within a given size.

The MASSIM concept and that of longer-term follow-on missions is closely related to that of the MAXIM mission ${ }^{43,44}$ which has been extensively studied. It builds on that work but identifies a way of achieving similar objectives that is in many respects simpler and less technologically demanding. The imaging techniques proposed for MASSIM are directly extendable to the (sub-) micro-arc-second scale needed for black hole imaging. Extension to the micro-arc-second regime is possible in either of two ways. First, the same lens concept will work for $\gamma$-rays - in fact both imaging and efficiency improve with energy and in addition more conventional materials such as polymers or aluminum can be used in place of beryllium. A $6 \mathrm{~m}$ lens working at $500 \mathrm{keV}$ will have a diffraction limit of 0.1 micro-arc-seconds. Secondly, the same angular resolution can be obtained in the region of the 6-7 $\mathrm{keV}$ Fe lines, using a partially filled aperture with diffractive lens components carried by multiple lens spacecraft spread over an effective lens diameter of $500 \mathrm{~m}$. Thus the X-ray optics to perform a black hole imaging mission is in principle available; only formation flying and pointing determination issues impede a direct step to this objective.

\section{CONCLUSIONS}

In the form proposed, MASSIM would be capable of making dramatic advances in X-ray astronomy, providing an angular resolution about three orders of magnitude beyond the current state of the art and allowing, for example, the ejection, acceleration and collimation processes taking part at the root of astrophysical jets to be examined in detail. The diffractive-refractive optics proposed for MASSIM has the potential for a further $\sim 3$ orders of magnitude improvement in angular resolution, allowing the goals of a 'Black Hole Imager' mission to be attained.

\section{ACKNOWLEDGMENTS}

We thank Jessie Leitner for helpful input concerning formation-flying and David Holdridge for invaluable help in preparation of the MASSIM proposal. The proposal was prepared with the aid of the NASA-GSFC Proposal Support Office.

\section{REFERENCES}

[1] Greco, V., Molesini, G., and Quercioli, F., "Telescopes of Galileo.," Appl. Opt. 32, 6219-6226 (Nov. 1993).

[2] Croton, D. J., Springel, V., White, S. D. M., De Lucia, G., Frenk, C. S., Gao, L., Jenkins, A., Kauffmann, G., Navarro, J. F., and Yoshida, N., "The many lives of active galactic nuclei: cooling flows, black holes and the luminosities and colours of galaxies," Mon. Not. R. Astr. Soc. 365, 11-28 (Jan. 2006).

[3] Sikora, M., Begelman, M. C., Madejski, G. M., and Lasota, J.-P., "Are Quasar Jets Dominated by Poynting Flux?," Astrophys. J. 625, 72-77 (May 2005).

[4] Marscher, A. P., Jorstad, S. G., Gómez, J.-L., Aller, M. F., Teräsranta, H., Lister, M. L., and Stirling, A. M., "Observational evidence for the accretion-disk origin for a radio jet in an active galaxy," Nature 417, 625-627 (June 2002).

[5] Merloni, A. and Fabian, A. C., "Coronal outflow dominated accretion discs: a new possibility for lowluminosity black holes?," Mon. Not. R. Astr. Soc. 332, 165-175 (May 2002). 
[6] Hujeirat, A., "A model for electromagnetic extraction of rotational energy and formation of accretionpowered jets in radio galaxies," Astron. E3 Astrophys. 416, 423-435 (Mar. 2004).

[7] Meier, D. L., "A Magnetically Switched, Rotating Black Hole Model for the Production of Extragalactic Radio Jets and the Fanaroff and Riley Class Division," Astrophys. J. 522, 753-766 (Sept. 1999).

[8] Blandford, R. D. and Znajek, R. L., "Electromagnetic extraction of energy from Kerr black holes," Mon. Not. R. Astr. Soc. 179, 433-456 (May 1977).

[9] Fender, R., Koerding, E., Belloni, T., Uttley, P., McHardy, I., and Tzioumis, T., "Eight powers of ten: similarities in black hole accretion on all mass scales," ArXiv e-prints 706 (June 2007).

[10] Junor, W., Biretta, J. A., and Livio, M., "Formation of the radio jet in M87 at 100 Schwarzschild radii from the central black hole," Nature 401, 891-892 (Oct. 1999).

[11] Reynolds, C. S., Fabian, A. C., Celotti, A., and Rees, M. J., "The matter content of the jet in M87: evidence for an electron-positron jet," Mon. Not. R. Astr. Soc. 283, 873-880 (Dec. 1996).

[12] Dunn, R. J. H., Fabian, A. C., and Celotti, A., "Using radio bubbles to constrain the matter content of AGN jets," Mon. Not. R. Astr. Soc. 372, 1741-1748 (Nov. 2006).

[13] Falcke, H., "The Silent Majority - Jets and Radio Cores from Low-Luminosity Black Holes," in [Reviews in Modern Astronomy], Schielicke, R. E., ed., Reviews in Modern Astronomy 14, 15-+ (2001).

[14] Brunthaler, A., Falcke, H., Bower, G. C., Aller, M. F., Aller, H. D., Teräsranta, H., Lobanov, A. P., Krichbaum, T. P., and Patnaik, A. R., "III Zw 2, the first superluminal jet in a Seyfert galaxy," Astron. \& Astrophys. 357, L45-L48 (May 2000).

[15] Narayan, R., Mahadevan, R., Grindlay, J. E., Popham, R. G., and Gammie, C., "Advection-dominated accretion model of Sagittarius $\mathrm{A}^{*}$ : evidence for a black hole at the Galactic center.," Astrophys. J. 492, 554-568 (Jan. 1998).

[16] Kennel, C. F. and Coroniti, F. V., "Confinement of the Crab pulsar's wind by its supernova remnant," Astrophys. J. 283, 694-709 (Aug. 1984).

[17] Weisskopf, M. C., Hester, J. J., Tennant, A. F., Elsner, R. F., Schulz, N. S., Marshall, H. L., Karovska, M., Nichols, J. S., Swartz, D. A., Kolodziejczak, J. J., and O’Dell, S. L., "Discovery of Spatial and Spectral Structure in the X-Ray Emission from the Crab Nebula," Astrophys. J. 536, L81-L84 (June 2000).

[18] Feigelson, E., "Introduction to X-rays Irradiation of Protoplanetary Disks," in [AAS/High Energy Astrophysics Division], AAS/High Energy Astrophysics Division 10, 17.01 (Mar. 2008).

[19] Herczeg, G. J., "Observational Diagnostics of X-ray-Irradiated Protoplanetary Disks," in [AAS/High Energy Astrophysics Division], AAS/High Energy Astrophysics Division 10, 17.02 (Mar. 2008).

[20] Kashyap, V., Drake, J., and Saar, S., "X-ray Activity and Close-in Giant Planets," in [AAS/High Energy Astrophysics Division], AAS/High Energy Astrophysics Division 10, 17.04 (Mar. 2008).

[21] Shu, F. H., "Astrophysics of X-ray Irradiated Protoplanetary Disks," in [AAS/High Energy Astrophysics Division], AAS/High Energy Astrophysics Division 10, 17.03 (Mar. 2008).

[22] Baez, A. V., "Historical description of selected experiments in the early development of x-ray optics," in [Proc. SPIE Vol. 1546, p. 472-488, Multilayer and Grazing Incidence X-Ray/EUV Optics, Richard B. Hoover; Ed.], Hoover, R. B., ed., Presented at the Society of Photo-Optical Instrumentation Engineers (SPIE) Conference 1546, 472-488 (Jan. 1992).

[23] Elwert, G., "X-Ray Picture of the Sun Taken with Fresnel Zone Plates," in [Structure and Development of Solar Active Regions], Kiepenheuer, K. O., ed., IAU Symposium 35, 439-+ (1968).

[24] Skinner, G. K., "Prospects for high angular resolution in gamma-ray astronomy," in [Galaxies and their Constituents at the Highest Angular Resolutions], Schilizzi, R. T., ed., IAU Symposium 205, 463-+ (Jan. 2001).

[25] Skinner, G. K., "Diffractive/refractive optics for high energy astronomy. I. Gamma-ray phase Fresnel lenses," Astron. ES Astrophys. 375, 691-700 (Aug. 2001).

[26] Gorenstein, P., "Concepts: x-ray telescopes with high-angular resolution and high throughput," in $[X$-Ray and Gamma-Ray Telescopes and Instruments for Astronomy. Edited by Joachim E. Truemper, Harvey D. Tananbaum. Proceedings of the SPIE, Volume 4851, pp. 599-606 (2003).], Truemper, J. E. and Tananbaum, H. D., eds., Presented at the Society of Photo-Optical Instrumentation Engineers (SPIE) Conference 4851, 599-606 (Mar. 2003). 
[27] Skinner, G. K., "Diffractive-refractive optics for high energy astronomy. II. Variations on the theme," Astron. Es Astrophys. 383, 352-359 (Jan. 2002).

[28] Skinner, G. and Gorenstein, P., "Astronomy (communication Arising): Black holes, fleas and microlithography," Nature 426, 245-246 (Nov. 2003).

[29] Braig, C. and Predehl, P., "X-ray astronomy with ultra-high-angular resolution," in [UV and Gamma-Ray Space Telescope Systems. Edited by Hasinger, Günther; Turner, Martin J. L. Proceedings of the SPIE, Volume 5488, pp. 601-612 (2004).], Hasinger, G. and Turner, M. J. L., eds., Presented at the Society of Photo-Optical Instrumentation Engineers (SPIE) Conference 5488, 601-612 (Oct. 2004).

[30] Skinner, G. K., von Ballmoos, P., Halloin, H., Gehrels, N. A., and Krizmanic, J. F., "Diffraction-limited gamma-ray imaging with Fresnel lenses," in [X-Ray and Gamma-Ray Telescopes and Instruments for Astronomy. Edited by Joachim E. Truemper, Harvey D. Tananbaum. Proceedings of the SPIE, Volume 4851, pp. 1366-1373 (2003).], Truemper, J. E. and Tananbaum, H. D., eds., Presented at the Society of Photo-Optical Instrumentation Engineers (SPIE) Conference 4851, 1366-1373 (Mar. 2003).

[31] Gorenstein, P., "Role of diffractive and refractive optics in x-ray astronomy," in [Optics for EUV, X-Ray, and Gamma-Ray Astronomy. Edited by Citterio, Oberto; O'Dell, Stephen L. Proceedings of the SPIE, Volume 5168, pp. $411-419$ (2004).], Citterio, O. and O'Dell, S. L., eds., Presented at the Society of Photo-Optical Instrumentation Engineers (SPIE) Conference 5168, 411-419 (Feb. 2004).

[32] Braig, C. and Predehl, P., "Large-scale diffractive X-ray telescopes," Experimental Astronomy 21, 101-123 (Apr. 2006).

[33] Braig, C. and Predehl, P., "Efficient Fresnel x-ray optics made simple," Appl. Opt. 46, 2586-2599 (May 2007).

[34] Skinner, G., von Ballmoos, P., Gehrels, N., and Krzmanic, J., "Diffraction limited gamma-ray optics using Fresnel lenses for micro-arc second angular resolution," in [Bulletin of the American Astronomical Society], Bulletin of the American Astronomical Society 35, 644-+ (Mar. 2003).

[35] Skinner, G. K., "Design and Imaging Performance of Achromatic Diffractive-Refractive X-Ray and GammaRay Fresnel Lenses," Appl. Opt. 43, 4845-4853 (Sept. 2004).

[36] Skinner, G. K., von Ballmoos, P., Gehrels, N., and Krizmanic, J., "Fresnel lenses for x-ray and gamma-ray astronomy," in [Optics for EUV, X-Ray, and Gamma-Ray Astronomy. Edited by Citterio, Oberto; O'Dell, Stephen L. Proceedings of the SPIE, Volume 5168, pp. 459-470 (2004).], Citterio, O. and O'Dell, S. L., eds., Presented at the Society of Photo-Optical Instrumentation Engineers (SPIE) Conference 5168, 459-470 (Feb. 2004).

[37] Gorenstein, P., "Diffractive-Refractive Optics for Higher Angular Resolution X-Ray Astronomy," in [Bulletin of the American Astronomical Society], Bulletin of the American Astronomical Society 205, 601-+ (Dec. 2004).

[38] Braig, C. and Predehl, P., "A diffraction limited dual-band x-ray telescope," in [Optics for EUV, X-Ray, and Gamma-Ray Astronomy III. Edited by O'Dell, Stephen L.; Pareschi, Giovanni. Proceedings of the SPIE, Volume 6688, pp. 668808 (2007).], Presented at the Society of Photo-Optical Instrumentation Engineers (SPIE) Conference 6688 (Sept. 2007).

[39] Gorenstein, P., Phillips, J. D., and Reasenberg, R. D., "Refractive/diffractive telescope with very high angular resolution for X-ray astronomy," in [Optics for EUV, X-Ray, and Gamma-Ray Astronomy II. Edited by Citterio, Oberto; O'Dell, Stephen L. Proceedings of the SPIE, Volume 5900, pp. 369-376 (2005).], Citterio, O. and O'Dell, S. L., eds., Presented at the Society of Photo-Optical Instrumentation Engineers (SPIE) Conference 5900, 369-376 (Aug. 2005).

[40] Morgan, B., Waites, C., Krizmanic, J., and Ghodssi, R., "Development of deep Silicon Phase Fresnel Lense using gray scale lithography and deep reactive ion etching," J. Microelectr. Sys. 13, 113 (2004).

[41] Krizmanic, J., Morgan, B., Streitmatter, R., Gehrels, N., Gendreau, K., Arzoumanian, Z., Ghodssi, R., and Skinner, G., "Development of ground-testable phase fresnel lenses in silicon," Experimental Astronomy 20, 299-306 (Dec. 2005).

[42] Luquette, R. and Sanner, R., "Spacecraft Formation Control: Managing Line-of-Sight Drift Based on the Dynamics of Relative Motion," in [Proceedings of the International Symposium on Formation Flying, The Netherlands, April 2008], ESA Special Publication SP-654 (2008). 
[43] Cash, W. C., "Maxim: micro-arcsecond x-ray imaging mission," in [Interferometry in Space. Edited by Shao, Michael. Proceedings of the SPIE, Volume 4852, pp. 196-209 (2003).], Shao, M., ed., Presented at the Society of Photo-Optical Instrumentation Engineers (SPIE) Conference 4852, 196-209 (Feb. 2003).

[44] Gendreau, K. C., Leitner, J., Markley, L., Cash, W. C., and Shipley, A. F., "Requirements and options for a stable inertial reference frame for a 100-micro-arcsecond imaging telescope," in [Interferometry in Space. Edited by Shao, Michael. Proceedings of the SPIE, Volume 4852, pp. 685-694 (2003).], Shao, M., ed., Presented at the Society of Photo-Optical Instrumentation Engineers (SPIE) Conference 4852, 685-694 (Feb. 2003). 